\title{
Experimental Study on Impact Loads Acting on Free-falling Modified Wigley ${ }^{\dagger}$
}

\author{
Sa Young Hong ${ }^{1 *}$, Young Shik Kim¹, Jo Hyun Kyoung ${ }^{2}$, Seok Won Hong ${ }^{1}$ \\ and Yong Hwan Kim ${ }^{3}$ \\ ${ }^{1}$ Maritime and Ocean Engineering Research Institute, KIOST, 171 Jang-dong, Yuseong-gu, Daejeon, Korea \\ ${ }^{2}$ Technip USA Inc., Houston, Texas, USA Department of Naval Architecture and Ocean Engineering, Seoul National University, \\ Seoul, Korea
}

(Manuscript Received June 17 2012; Revised July 28, 2012; Accepted August 5, 2012)

\begin{abstract}
The characteristics of an impact load and pressure were experimentally investigated. Drop tests were carried out using a modified Wigley with $\mathrm{CB}=0.56$. The vertical force, pressures, and vertical accelerations were measured. A 6-component load cell was used to measure the forces, piezo-electric sensors were used to capture the impact pressure, and strain-gauge type accelerometers were used to measure the vertical accelerations. A 50-kHz sampling rate was applied to capture the peak values.

The repeatability of the measured data was confirmed and the basic characteristics of the impact load and pressure such as the linearity to the falling height were observed for all of the measurements. A simple formula was derived to extract the physical impact load from the measured force based on a simple mass-sensor-mass diagram, which was validated by comparing impact forces with existing data using the mathematical model of Faltinsen and Chezhian (2005). The effects of the elasticity of the model and change in acceleration during the water entry were investigated. It is interesting to observe that the impact loads occurred and reached peak values at the same time duration after water entry for all drop heights.
\end{abstract}

Keywords: Impact Pressure, Impact loads, Slamming, Free-Falling Drop Test, Modified Wigley

\section{Introduction}

Sea-going vessels and various kinds of onshore and off-shore structures have frequently experienced impact forces such as slamming. The impact force on a ship caused by slamming induces significant structural stress and vibrations, which can lead to the local or global failure of the structure. The importance of more accurate and reliable estimations of slamming loads has been increasing, not only for designing structurally safe ships, but also for ensuring the survivability of marine structures under harsh environments.

\footnotetext{
† This paper was presented at the International Conference on Violent Flows (VF 2007), Fukuoka, Japan, November 2007.

"Corresponding author. Tel.: +82 42-866-3930, Fax.: +82 42-866-3919, E-mail address: sayhong@kisot.ac

Copyright (C) KSOE 2012.
}

Remarkable researches on slamming loads were conducted by Von Karman [1] and Wagner [2], which still provide the fundamentals of our physical understanding and intuition on slamming. Experimental researches followed their pioneering researches, most of which treated simple geometries such as two-dimensional sections of cylinders and wedges. A study on the numerical prediction of three-dimensional slamming was attempted by Troesch and Kang [3] for flare slamming. Chuang $[4,5]$ investigated the three-dimensional effect by considering the effect of an air cushion trapped on a wet body surface and using model tests for a conical shaped body. Recently, Chezhian [6], and Faltinsen and Chezhian [7], carried out model tests and numerical analyses for a fore and aft symmetric three-dimensional body combined with a cylinder 
and hemi-sphere. Faltinsen [8] adopted the matched asymptotic expansion method to consider the water entry of a wedge, Korobkin [9] derived analytical models for the water impact problem. Yettou et al. [10] derived a new analytical model that considered the change in the water entry velocity caused by deceleration, following the work done by Mei et al. [11].

Most of the numerical works have been based on the following assumptions: a rigid body, incompressibie and inviscid fluid, irrotational flow, calm water, neglect of gravity and air trapping, and constant water entry velocity. Accordingly, the experimental works have focused on validating the state of the art numerical and analytical models. Consequently, the models have been made so rigid and heavy that the conventional assumptions on slamming or impact are satisfied. However, ships suffer slamming in the following real situations: they experience acceleration by gravity and deceleration by a reaction force after impact, and the slamming induces very significant hydroelastic vibrations, which means the ship is not sufficiently rigid to be regarded as a rigid body. Thus, it is meaningful to investigate the results of an impact model test with less idealization, allowing changes in the acceleration, velocity, and elasticity of the model.

In the present study, the characteristics of global slamming loads, as well as the pressure on a freely falling Wigley hull with $\mathrm{CB}=0.56$, were investigated experimentally. A high speed digital camcorder was used for observing the violent flow around a ship model.

The basic characteristics of the impact load and pressure such as the linearity to the falling height were observed for all of the measurements. A simple formula was derived to extract the real impact load from the measured force based on a simple mass-sensor-mass diagram.

It was found that the time durations required for the impact loads to reach their peak values were the same for different drop heights, which corresponded to the time needed to develop a splash after touching the undisturbed free surface. It was also found that the elasticity of the impact body significantly influenced the level of slamming load, with a more elastic body giving a much higher slamming load than a rigid body.

\section{Model Tests}

\subsection{Test Setup and Model}

The model tests were carried out at the preparation tank of the MOERI Ocean Engineering Basin. The flume is $5 \mathrm{~m}$ long, $3 \mathrm{~m}$ wide, and $2 \mathrm{~m}$ deep. The water depth was kept at $1.4 \mathrm{~m}$ for the tests. The test setup was devised to prevent the friction effect during the free drop of the model (see Fig. 1). The mass distribution of the model was made uniform to prevent any model inclination from non-uniformity. To maintain a horizontal posture at the beginning stage of the free drop when triggering via a pneumatic valve, a guide frame made of four vertical steel poles and conical wedges was employed. This guide frame structure made it possible for the model to freely drop without any interference. The sample record of measured accelerations shown in Fig. 2 shows no contact between the model and guide frame structure. Recorded video scenes at the moments the model touched the still water surface also show that the model maintained its initial horizontal posture. The model was a modified Wigley hull with $\mathrm{CB}=0.57$ made of FRP (fiberglass reinforced plastic). The mathematical description of the hull form is as follows.

$$
\begin{aligned}
& \eta=\left(1-\xi^{2}\right)\left(1-\xi^{2}\right)\left(1+0.2 \xi^{2}\right)+\xi^{2}\left(1-\xi^{8}\right)\left(1-\xi^{2}\right)^{4} \\
& \xi=2 x / L, \eta=2 y / B, \xi=z / d \\
& -L / 2 \leq x \leq L / 2,-d \leq z \leq 0 \\
& L \times B \times d=1.25 m \times 0.25 m \times 0.25 m
\end{aligned}
$$

A stiffener structure made of aluminum was installed along the bottom of the model to suppress any unwanted elastic deck motion effect on the impact force and accelerations. A load cell was installed at the top of the stiffener structure to measure the impact force on the model caused by its free drop. The weight of the Wigley hull model itself was $36.7 \mathrm{kgf}$; the total weight of the model, including the sensors, sensor frame, and additional weights, was $149.4 \mathrm{kgf}$. The falling heights were set to range from $0.1 \mathrm{~m}$ to $0.5 \mathrm{~m}$ in $0.1 \mathrm{~m}$ increments. At least three identical tests were carried out, and the repeatability of the data was confirmed.

Table 1 Sensor characteristics.
\begin{tabular}{|l|l|l|}
\hline Items & Sensors & $\begin{array}{c}\text { Natural } \\
\text { frequency }\end{array}$ \\
\hline Force & $\begin{array}{l}\text { ATI OMEGA 190 SI- } \\
7200-1400\end{array}$ & $1.3 \mathrm{KHz}$ \\
\hline Accelerations & KYOWA AS-20HB & $1.3 \mathrm{KHz}$ \\
\hline Pressure & $\begin{array}{l}\text { KISTLER 710A, } \\
\text { Piezo-electric }\end{array}$ & $70 \mathrm{KHz}$ \\
\hline
\end{tabular}




\subsection{Sensors and measurements}

The impact force, pressures at 7 locations, and vertical accelerations on the bottom and deck of the model and the mounting plate were measured. The sampling rate for all of the measurements was set to $50 \mathrm{kHz}$ to capture the impact characteristics. A PXI-4472 system from National Instrument was employed for data acquisition. A MotionProHs-2 was used to record the violent flow around the model during the tests at 500 frames/second. The sensor locations are shown in Fig. 3. The specifications of the sensors are summarized in Table 1.

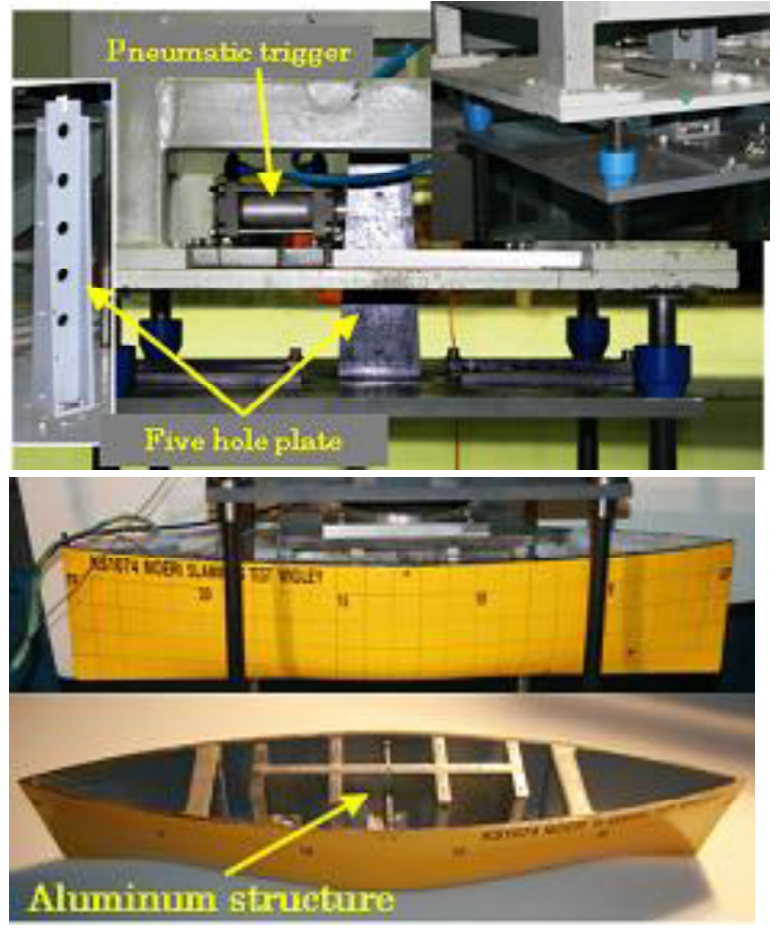

Fig. 1 Photos of test setup and model.

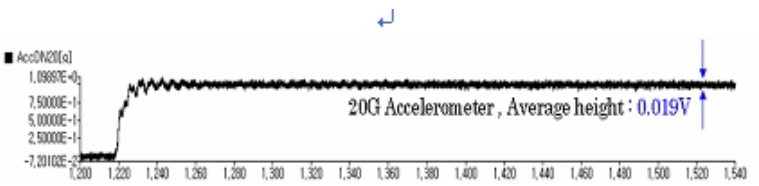

Fig. 2 Acceleration signal during free drop.

\subsection{Impact Load Measurement and Analysis}

Because the load cell was located between the ship hull and mounting plate, the measured impact force was influenced by the mass distribution of the hull and mounting plate. In order to extract the pure impact force from the measured value, the model system was idealized as a two lumped mass system connected with a rigid load cell, as shown in Fig. 4,

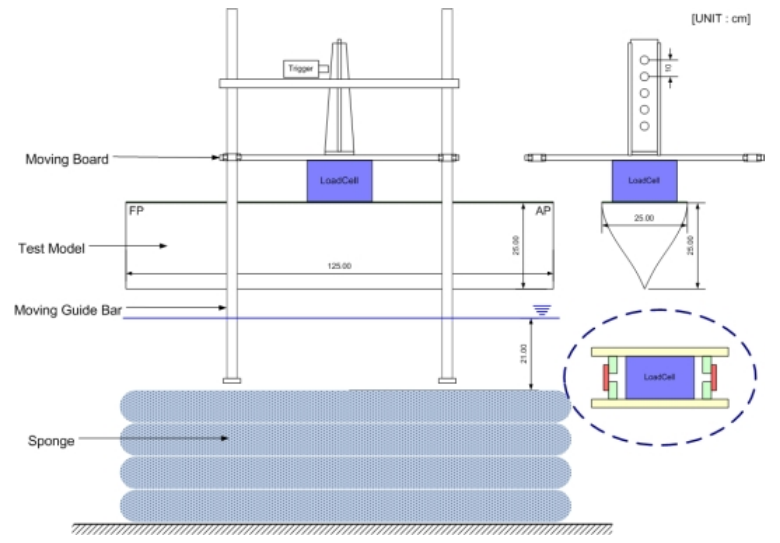

Fig. 3 Sensor locations.

and the equation of motion was derived to extract the impact load from the measured force.

$$
\begin{aligned}
& m_{1} a_{\text {sig }}=f_{\text {sig }}+m_{2} g+m_{1} g \\
& m_{2} a_{\text {sig }}=-f_{\text {sig }}-m_{2} g+m_{2} g+R_{f}
\end{aligned}
$$

where $a_{\text {sig }}$ and $f_{\text {sig }}$ are the measured acceleration and force, respectively. The impact force can be expressed by the following three equations from the relationship between equations (2) and (3).

$$
\begin{aligned}
& R_{f 1}=-f_{\text {sig }}-m_{2} a_{s i g} \\
& R_{f 2}=\left(m_{1}+m_{2}\right)\left(g-a_{s i g}\right) \\
& R_{f 3}=-\frac{m_{1}+m_{2}}{m_{1}}\left(f_{s i g}+m_{2} g\right)
\end{aligned}
$$
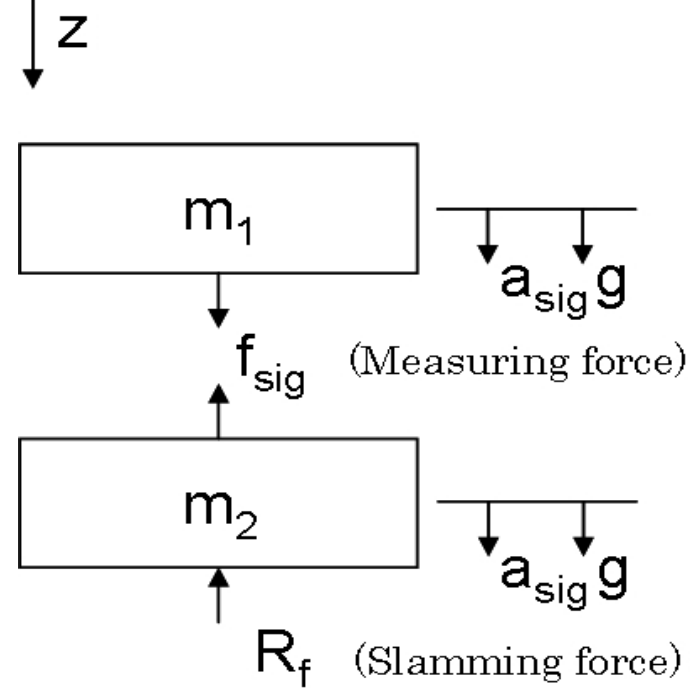

Fig. 4 Two body diagram of free drop system. 
The accelerations measured at different positions such as the bottom of the ship model and top of the mounting plate show similar behaviors (Fig. 5). The general description of the mass-spring-damper system for the model system is simplified as a two lumped mass system connected with a load cell in the present paper.

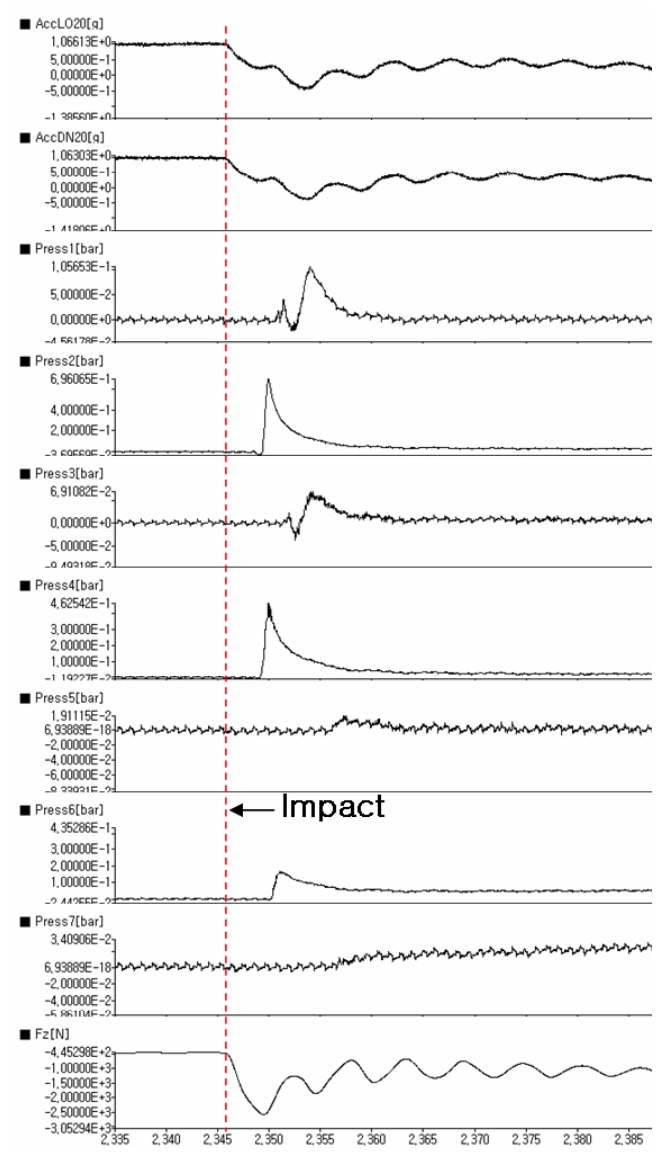

Fig. 5 Set of acquisition signals at $\mathrm{h}=0.5 \mathrm{~m}$.

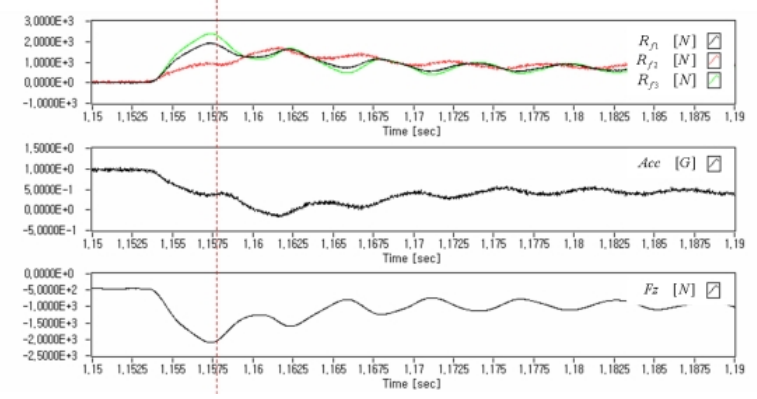

Fig. 6 Raw signal for measured vertical force (Fz) and slamming forces (Rf1,2,3_Fz) analyzed by mathematical formulation.
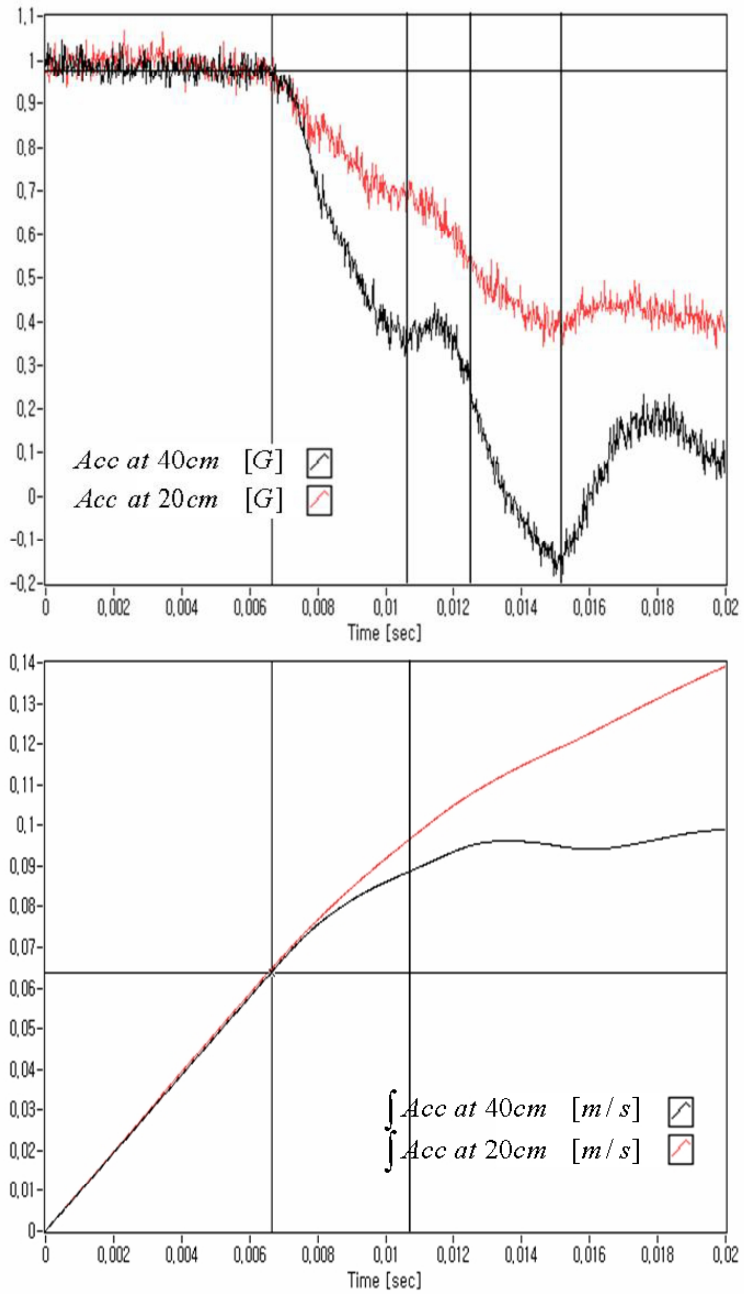

Fig. 7 Acceleration and velocity of model during water en$\operatorname{try}(\mathrm{h}=0.2 \& 0.4 \mathrm{~m})$.

\section{Results and Discussion}

Fig. 5 shows a typical example of the measured signal from the model tests. The accelerations and vertical force concurrently react at the moment of impact, and the pressure signals follow with intervals corresponding to their locations. It can be clearly seen that the acceleration changes dramatically during the very short time interval of impulsive water entry.

This fact implies that the body suffers from a significant change in acceleration during the impact. Fig. 7 presents the change in acceleration and the falling velocity of the model during the water entry. The velocity increases linearly until it touches the free surface, after which the velocity increase rate becomes smaller as a result of deceleration. In actuality, the entry velocity still increases until the ship 
suffers the peak load caused by slamming, even if the ship experiences a deceleration because of the fluid reaction force. In the model experiment, the velocity changes during the impact are about $1 \%$ and $2 \%$ of the entry velocity for drop heights of 0.4 $\mathrm{m}$ and $0.2 \mathrm{~m}$, respectively. This means that a constant velocity assumption may slightly underestimate the slamming load for smaller entry velocities, but not very significantly.

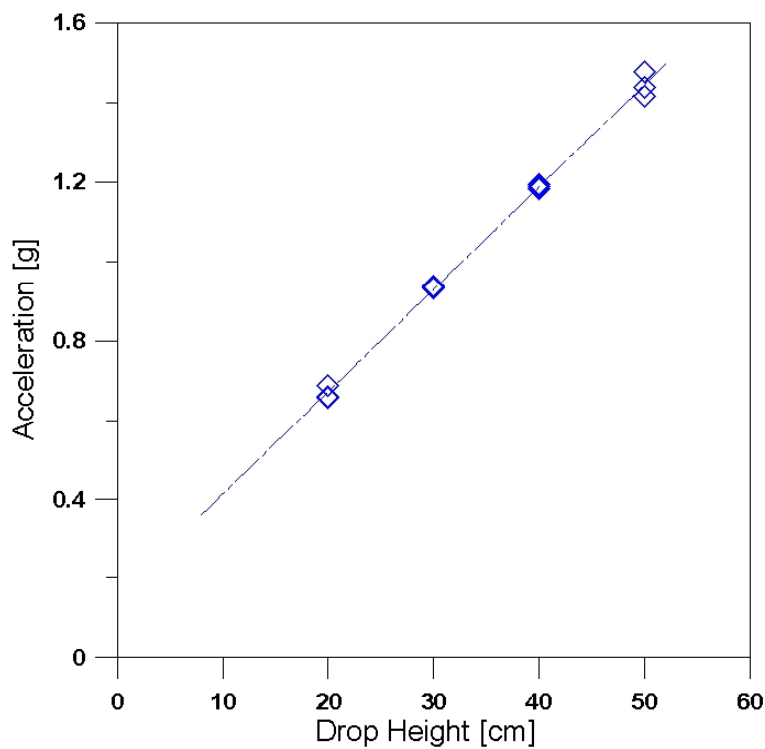

(a) Maximum acceleration

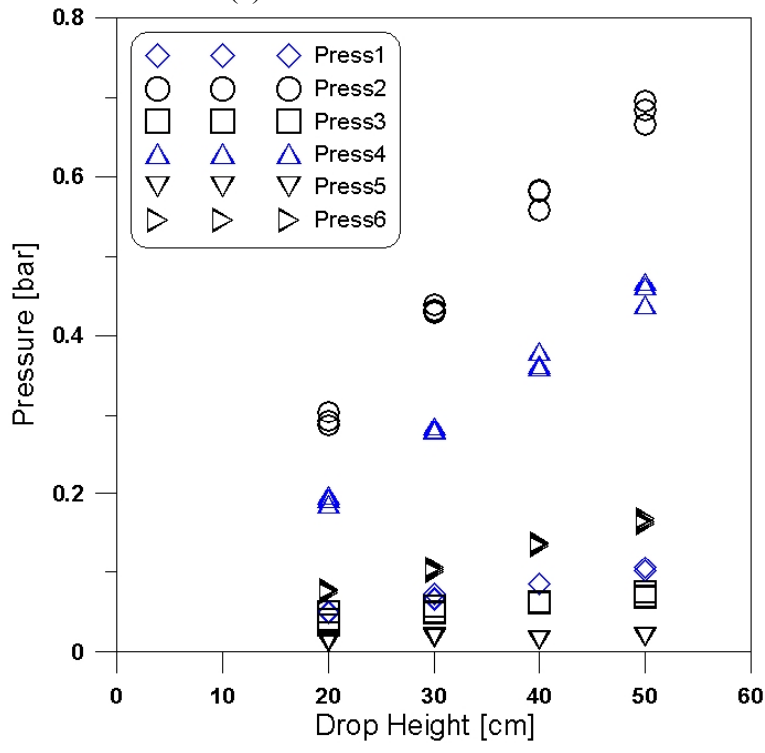

(b) Maximum pressure

Fig. 8 Maximum accelerations and pressures at various drop heights.

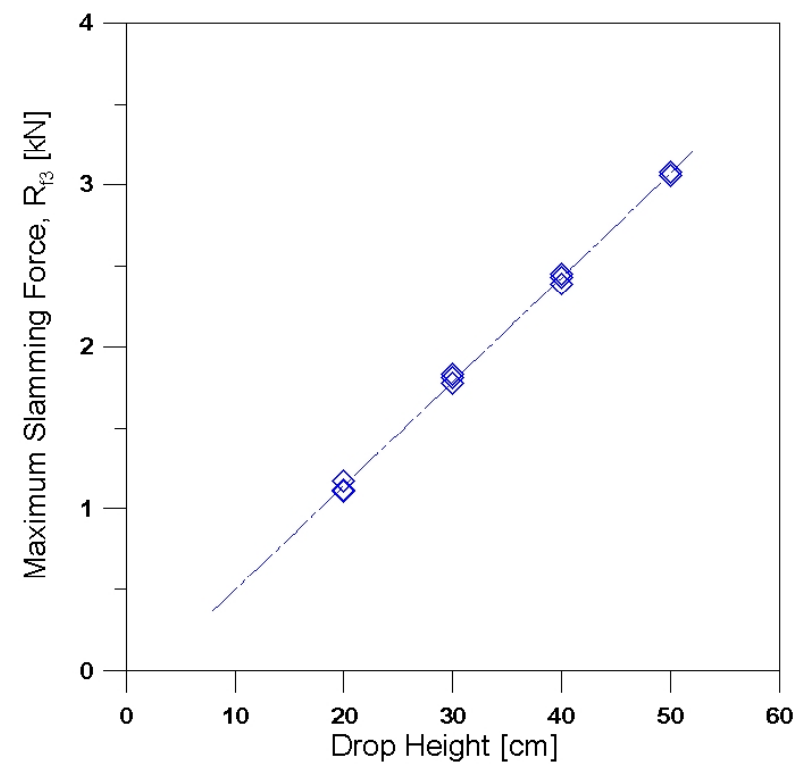

(a) Maximum slamming force(Rf3)

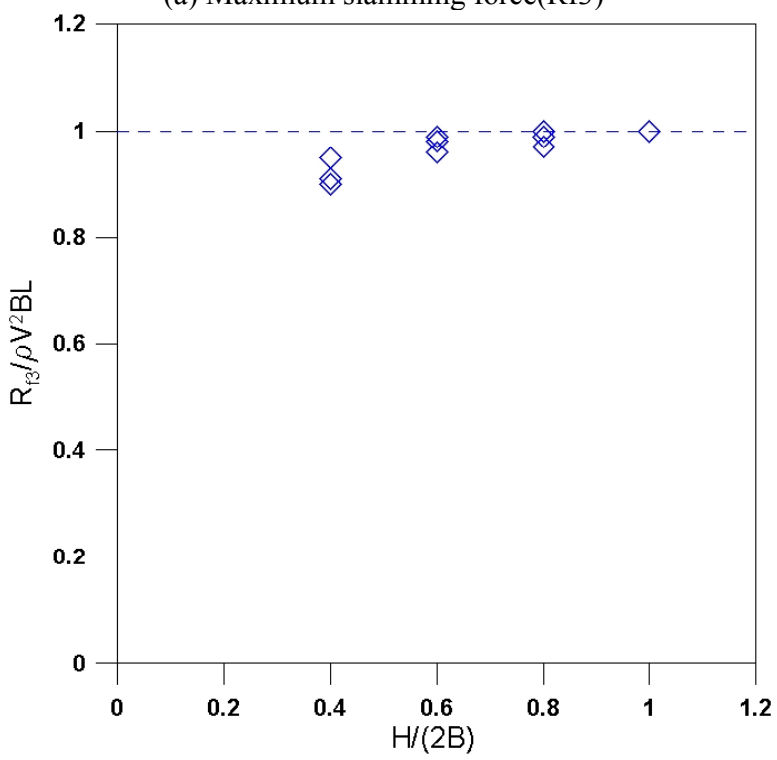

(b) Nondimensionalized slamming force (Rf3/pV2BL) with nondimensional drop height $(\mathrm{H} / 2 \mathrm{~B})$.

Fig. 9 Characteristics of slamming force for different drop heights.

Fig. 8 shows the relationship between the measured peak acceleration values, pressures, and drop heights. The acceleration and pressures are linearly proportional to the drop height, which shows that the impact force is proportional to the square of the entry velocity. However, a pressure with a very low level such as the one at location 5 does not show a clear linear relation to the drop height because pressure 5 is not primarily induced by the impact. It is also observed that the test results show satisfactori- 
ly good repeatability for 3 tests under the same conditions. Both the repeatability results and the linearity of the impact values of the force and pressure show the qualitative reliability of the measurements in the model tests.

The impact force is derived from the measured vertical force using equations (4a 4c)(Fig. 8). Because the bottom of the load cell is mounted on the deck frame of the Wigley model and the top is bolted to a heavy upper plate, the measured force is a reaction force between the upper and lower parts of the ship model system. This reaction force is a small portion of the impact force ( ). Equation (4c) gives a reasonable correction for the measured value to obtain a more realistic impact load among the three different expressions for converting the measured value to a slamming load. In contrast, equations $(4 \mathrm{a} \& 4 \mathrm{~b})$ show different behaviors because they do not account for the effects of the mass distributions.

Fig. 9(a) shows that the impact load is proportional to the drop height. Consequently, the impact forces non-dimensionalized by the square of the entry velocity ( ) are constant for a drop height, as shown in Fig. 9(b). This result qualitatively coincides with that of Von Karman [1], which showed that the impact force at the initial entry was proportional to the square of the entry velocity. In our study, the magnitude of the normalized slamming force was about 1 , but the factor was dependent on the sectional shape and elastic property of the structure.

Fig. 10 shows the effect of the elasticity of the model on the impact load. The model was the same as that of Chezhian [6], and Faltinsen and Chezhian [7], but the weight and elasticity were different in the present study. The upper curve was obtained from the lighter and more elastic model (weight $=$ $143 \mathrm{kgf}$ ), while the lower curve was obtained from the heavier (168 kgf) and stiffened model. Chezhian [6] already discussed the effect of the model elasticity and sensor system on the slamming load and reviewed the difficulties encountered in previous experimental studies. It was observed that the elasticity of the model contributed to an amplification of the impact force, which means the elasticity of the ship should be considered for a safe ship design against slamming loads.

Fig. 11 shows the measured slamming force and those derived from the mathematical model studied by Chezhian [6], and Faltinsen and Chezian [7]. In the figure, the diamond and circular symbols denote the experimental and numerical values by Chezhian [6], while the square and triangular symbols are the measurements in the present study. The square symbol corresponds to a lighter and more elastic model, and the triangle corresponds to a heavier and stiffened model. The elastic model gives a higher slamming force, but the stiffened model shows good correlation with the calculations, as well as the measurements by Chezian [6].

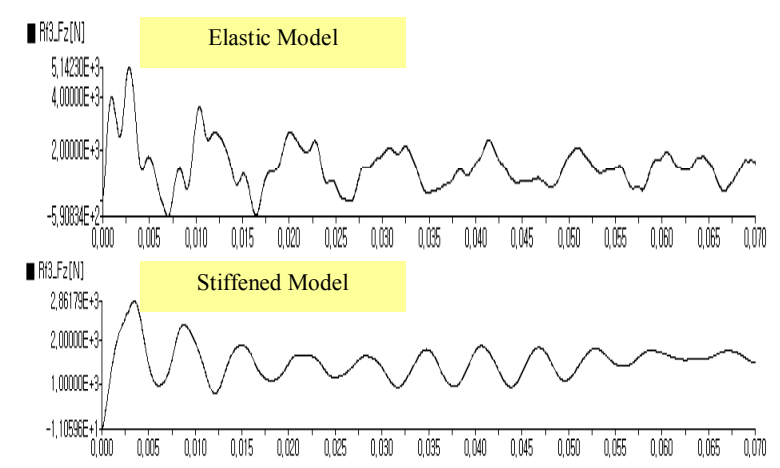

Fig. 10 Comparison of impact forces for different elastic properties of mathematical model (Chezhian, 2003).

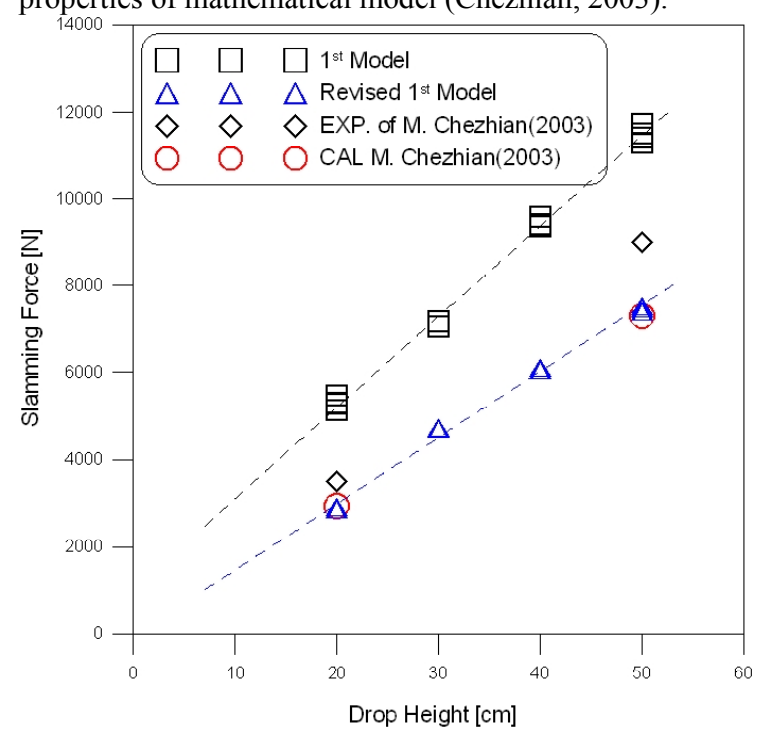

Fig. 11 Comparison of slamming forces for mathematical model (Chezhian(2003).

Fig. 12 shows snapshots of the model at the moment of water contact to 0.03 seconds after the water entry for the case of a drop height of $0.5 \mathrm{~m}$. It is observed that a splash occurs after the impact from the midship and develops both upward and in the fore and backward directions. 
Fig. 13 shows snapshots for the same case, with the time step reduced to $2 \mathrm{~ms}$ (milliseconds). It is observed that the splash caused by the water entry of the model develops at $t=6 \mathrm{~ms}$. Fig. 14 shows the measured impact force signals for different drop heights. It can be seen that peaks in the impact force appear at the same time $(\mathrm{t}=4 \mathrm{~ms})$ after the water entry for all drop heights. According to von Karman's and Wagner's formulas based on a constant drop velocity, the maximum impact force occurs at $\mathrm{z}=-0.018 \mathrm{~m}$, independent of the drop height. The corresponding times for the peak occurrences for heights of $0.2 \sim 0.5 \mathrm{~m}$ are estimated to be 5.8 9 $\mathrm{ms}$. The measured impact peak value appears earlier than the estimated one, which is based on a constant drop speed. The measured water entry velocities show relative errors of just $1 \sim 2 \%$. However, the locations where the peak force occurs are roughly estimated as $\mathrm{z}=-0.008 \sim-0.013 \mathrm{~m}$ for $\mathrm{h}=0.2 \sim 0.5 \mathrm{~m}$, which are shallower than the numerical predictions. It is not clear why the measured peaks appear at the same time after the water entry, but as shown in Fig. 14 , the splash develops at around $6 \mathrm{~ms}$ after water entry. The same phenomena were observed for other drop heights. The corresponding time $(4 \mathrm{~ms})$ at which the peak occurs seems to shows a close relationship with the time the splash develops after the water entry from the high-speed video record. The elasticity of the model might produce a high peak behavior similar to that of the impacts from different drop heights. Another possibility is that the model suffers a rapid change in acceleration during the water entry, which does not have a significant effect on the velocity change because of the very short time duration and effect of gravity, but could significantly influence the magnitude and peak time of the impact force by adding a force proportional to acceleration. The amount of acceleration dependent impact force is roughly estimated to be about $30 \%$ of the total impact force, which can lead to an earlier impact peak time compared to that under a constant velocity situation.

Fig. 15 compares the measured impact force and simple calculation results based on von Karman's and Wagner's theories. In the case of von Karman's theory, the impact force at the midship section of the modified Wigley can be obtained as a closed form by assuming a constant entry velocity.
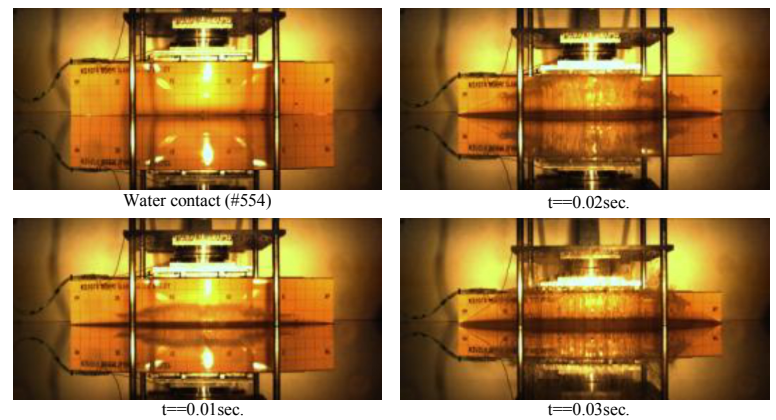

Fig. 12 Snapshots of water entry during 0.03 seconds $(h=0.5$ $\mathrm{m})$
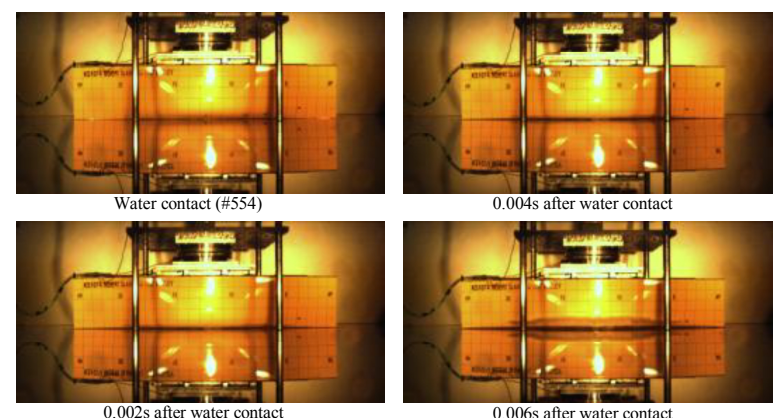

Fig. 13 Snapshots of water entry during 0.006 seconds $(h=$ $0.5 \mathrm{~m})$.

$F_{3}=-\frac{5 \pi \rho B c U^{2}}{d}\left(\frac{z}{d}\right)^{2}$

where $\mathrm{U}$ is the entry velocity, B is the breadth, $d$ is the draft, $\mathrm{c}$ is the instantaneous wetted breadth, and $\mathrm{z}$ is the vertical coordinate.

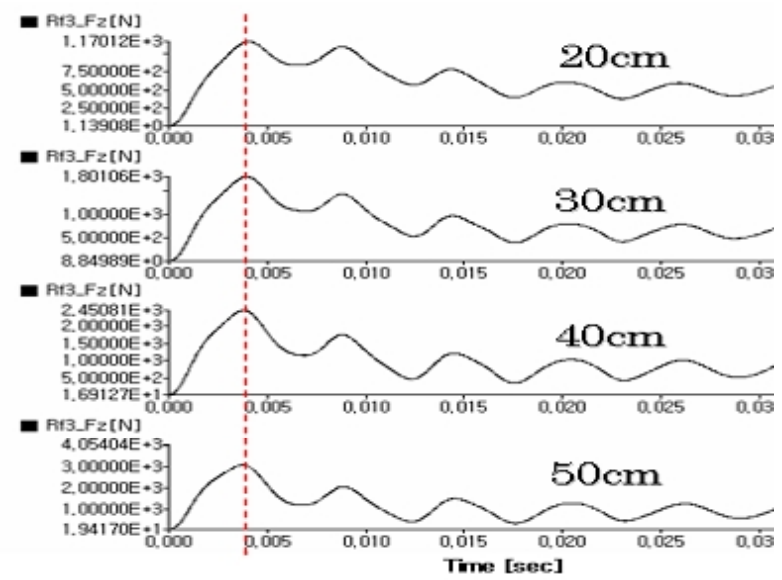

Fig. 14 Slamming force signals for different drop heights.

For the other sections, cross sections are approximated by polynomials up to the third-order. Then, the sectional impact forces are approximated based 
on the fitted polynomials. The same procedures are adopted for the calculation of Wagner's formula. In Fig. 14, the magnitudes of the calculated values are obtained by integrating along the length in a strip sense.

Both numerical results show qualitatively good correlation with the measurements, but the slopes are different. It is easily expected that von Karman's formula gives a lower bound for the impact force, while Wagner's method gives a kind of upper bound. Considering the discrepancies in the impact peak times, as well as other factors neglected in those formulas such as the 3-dimensional effect and general shape of the sections, the simply calculated values seem to give a good approximation in an engineering sense.

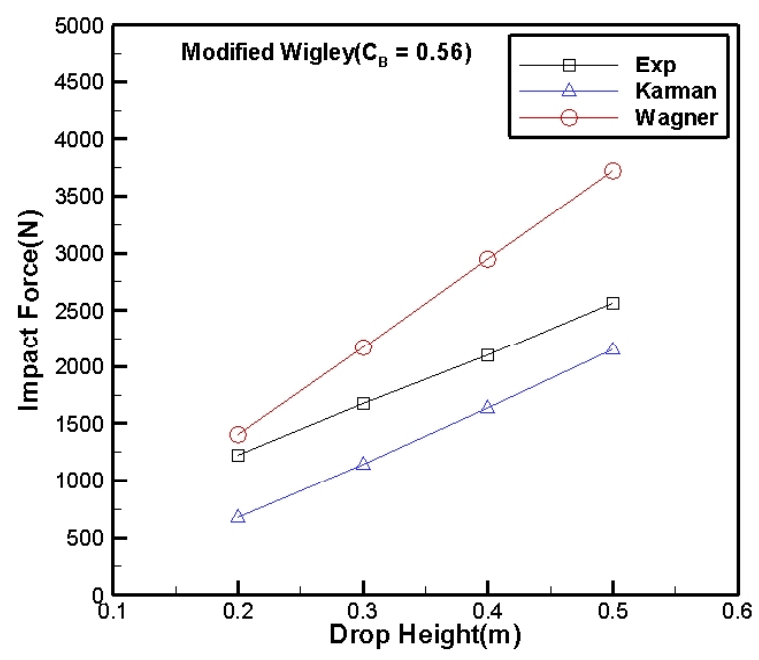

Fig. 15 Comparison of measured impact forces with numerical estimations.

\section{Conclusion}

An experimental study on slamming loads for a modified Wigley was carried out using free falling drop tests. The reliability of the measured data was confirmed by investigating the basic characteristics of the slamming loads such as the linear dependency on the drop height and the repeatability. Findings from the observations and analysis of the experimental data are summarized as follows:

- For most of the impact signal measurements, corrections should be made based on a reasonable physical model. An appropriate modeling was derived for extracting the slamming force from the measured impact force in the present study.

- The time duration to reach the peak impact force was observed to be the same for different drop heights, which had a close correlation with the observation that the time to develop the splash after water entry was the same for different drop heights. In the present study, the time at the peak force was 0.004 seconds, while the time the splash occurred was less than 0.006 seconds.

- The measured impact forces were very sensitive to the elasticity of the model. A more elastic body gave a much higher slamming force. This implies that a consideration of the elasticity of a ship's structure is needed for a more accurate and conservative estimation of the slamming load.

It was also observed that the acceleration of a freefalling body suffered a significant change in acceleration at the moment of impact, which could contribute to an earlier impact appearance time. The velocity change during the water entry was not significant, but was only a relative error of a few percent compared to the entry velocity.

\section{Acknowledgement}

This paper represents a portion of the results for "Studies on Three-dimensional Slamming on Slender Ships - Experimental Measurement of Impact Loads" funded by the Office of Naval Research. This study was also partially supported by the basic research program "Development of Safety Evaluation Technologies for Marine Structures in Disastrous Ocean Waves" funded by the Korea Research Council of Public Science \& Technology.

\section{References}

[1] [1] von Karman, T. (1929) "The Impact of Seaplane Floats During Landing," NACA TN321, Washington, DC

[2] Wagner, H. (1932) "Uber stoss-und Gleitvorgange ander Oberflache von Flussikeiten," Zeitschr. f. Angew. Math. Und. Mech., Vol 12, No 4, pp 193-235 
[3] Troesch, A.W. and Kang, C.G. (1988) "Evaluation of impact loads associated with flare slamming," SNAME, 3rd IMSDC, Pennsylvania, pp.117-132.

[4] Chuang, S. L. (1966) "Experiments on FlatBottom Slamming," Journal of Ship Research, Vol 10, pp 10-17

[5] Chuang, S. L. and Milne, D. T. (1971) "Drop Test of Cones to Investigate the ThreeDimensional Effects of Slamming," NSRDC Report 3543

[6] Chezhian, M.(2003) "Three-dimensional Analysis of Slamming," Doctoral Thesis, Norwegian Univ. of Science and Technology

[7] Faltinsen, O. M. and Chezhian, M. (2005) "A Generalized Wagner Method for Three-
Dimensional Slamming," Journal of Ship Research, Vol. 49, No. 4, pp.279-287

[8] Korobkin, A. (2004) "Analytical Models of Water Impact," Euro Jnl. of Applied Mathematics, vol. 15, pp821-838

[9] Faltinsen, O.M. (2002) "Water Entry of a Wedge with Finite Deadrise Angle," Journal of Ship Research 46, pp39-51

[10]Yettou, E-.M., Desrochers, A. and Champoux, Y. (2007) "A New Analytical Model for Pressure Estimation of Symmetrical Water Impact of a Rigid Wedge at Variable Velocities," Journal of Fluids and Structures 23, pp501-522

[11]Mei, X., Liu, Y. and Yue, D.K.P. (1999) "On the Water Impact of General Two-dimensional Sections," Applied Ocean Research, 21, pp1-15 